\title{
Produção científica e formação de recursos humanos em Bioquímica no Rio Grande do Sul, Brasil
}

\section{Scientific Production and Human Resources Training in Biochemistry in Rio Grande do Sul, Brazil}

\section{Producción científica y formación de recursos humanos en Bioquímica en Rio Grande do Sul, Brasil}

Luciana C. Berti, doutora em Educação em Ciências pela Universidade Federal do Rio Grande do Sul. Endereço: Rua Ramiro Barcellos, 2600, anexo. Departamento de Bioquímica da UFRGS. CEP: 90035-003 - Porto Alegre, RS. Telefone: (51) 3308-5544/ 5558. E-mail: luciana.berti@ufrgs.br.

Suelen Baggio, graduada em Ciências Biológicas pela Universidade Federal do Rio Grande do Sul e bolsista de Iniciação Científica do CNPq. Endereço: Rua Ramiro Barcellos, 2600, anexo. Departamento de Bioquímica da UFRGS. CEP: 90035-003 - Porto Alegre, RS. Telefone: (51) 3308-5544/ 5558. E-mail: suzy-gfbp@ hotmail.com.

Diogo O. Souza, doutor em Bioquímica pela Universidade Federal do Rio de Janeiro. Endereço: Rua Ramiro Barcellos, 2600, anexo. Departamento de Bioquímica da UFRGS. CEP: 90035-003 - Porto Alegre, RS. Telefone: (51) 3308-5544/ 5558. E-mail: diogo@ufrgs. br.

Susana T. Wofchuk, doutora em Bioquímica pela Universidade Federal do Paraná. Endereço: Rua Ramiro Barcellos, 2600, anexo. Departamento de Bioquímica da UFRGS. CEP: 90035-003 - Porto Alegre, RS. Telefone: (51) 3308-5544/ 5558. E-mail: swofchuk@ ufrgs.br. 


\section{Resumo}

A produção científica brasileira em Bioquímica está crescendo, e o Rio Grande do Sul se destaca nesse contexto. Por essa razão, o objetivo deste estudo é caracterizar o perfil dos professores/ pesquisadores em Bioquímica das IES públicas e privadas do RS, quantificar e analisar sua produção científica (número de artigos científicos publicados, dissertações e teses orientadas). Observamos que a maioria dos professores/pesquisadores em Bioquímica de todas as instituições, públicas ou privadas, é titulada e pós-graduada, mas nem todos conseguem seguir atividades de pesquisa aliadas àquelas de ensino nas instituições que os acolhem.

Palavras-chave: Cientometria. Bioquímica. Produção Científica.

\section{Abstract}

Brazilian scientific production in Biochemistry increased in recent years, and the state of Rio Grande do Sul (RS) presented significant relevance in this context. The objective of this study is to characterize the profile of researchers in Biochemistry of public and private universities of RS and to quantify and analyze their scientific production (scientific papers; Ms and PhD theses). We observed that the majority of the researchers in Biochemistry in public and private institutions have a PhD degree. However, various researchers do not develop research activities at the institutions where they are working.

Keywords: Scientometrics. Biochemistry. Scientific Production.

\section{Resumen}

La producción científica de Brasil en Bioquímica está creciendo y el estado de Rio Grande do Sul (RS) se sobresale en este contexto. Por esta razón, el objetivo de este estudio es caracterizar el perfil de los profesores/investigadores en Bioquímica de las instituciones de 
enseñanza superior públicos y privados del RS, cuantificar y analizar la producción científica (número de artículos científicos publicados, disertaciones y tesis supervisadas). Hemos observado que la mayoría de los profesores/investigadores en Bioquímica de todas las instituciones, públicas o privadas, es titulada y con posgrado, pero no todos logran conciliar las actividades de investigación con las de enseñanza en las instituciones educativas que los reciben.

Palabras clave: Cienciometría. Bioquímica. Producción Científica.

\section{Introdução}

A ciência pode ser considerada um mundo de ideias em movimento - o processo para a produção do conhecimento - e busca descobrir a unidade existente nas diferentes facetas da experiência do homem com o seu meio (ZANCAN, 2002; RUTHERFORD; ALGREEN, 1990). Assim, o desenvolvimento científico é um fator crucial para o bem-estar social a tal ponto que a distinção entre povos ricos e pobres é hoje feita pela capacidade de criar ou não conhecimento científico. Sem instituições adequadas de educação superior em ciência e tecnologia e em pesquisa, com uma massa crítica de cientistas experientes, nenhum país pode ter assegurado um desenvolvimento real (UNESCO, 2000).

O avanço explosivo do conhecimento está marginalizando os povos que não dispõem de uma infraestrutura de pesquisa associada à formação de recursos humanos de alto nivel e à uma educação científica universal. A análise da situação do Brasil mostra a necessidade da expansão da base de pesquisa acadêmica e da inovação tecnológica e destaca a urgência na mudança dos sistemas de ensino fundamental, médio e superior, passando de informativo para formativo, como meio de capacitação do homem para o mercado de trabalho, altamente dependente de um aprender contínuo (ZANCAN, 2002).

O processo de produção do conhecimento no Brasil sempre esteve ligado ao crescimento da pós-graduação (MENDES, 1991; LEITE 
et al., 2001). O País busca, principalmente por meio dos Programas de Pós-Graduação (PPG), consolidar sua base científica e formar recursos humanos capacitados para solucionar problemas regionais e nacionais. Um objetivo importante do sistema de formação é habilitar pesquisadores que possam cumprir tais objetivos. Esses produtores/difusores de conhecimento deverão ter domínio do estado de conhecimento na área em que atuam e capacidade de originar questões coerentes e atualizadas com o domínio metodológico para estudá-las. Com a estruturação de linhas de pesquisa autóctones, tornam-se multiplicadores na formação de novos pesquisadores, e a produção originada deve ser referendada por avaliadores externos do contexto nacional e internacional (UNESCO, 2005).

A provisão de ensino superior no Brasil é, atualmente, assegurada por Instituições de Ensino Superior (IES) públicas e privadas, com predomínio numérico das últimas.

O número de instituições públicas cresceu 3,8\% de 2008 para 2009, enquanto o número de instituições privadas cresceu 2,6\%. Apesar de ambas apresentarem a mesma tendência, as instituições privadas continuam predominantes na educação superior, com 89,4\% do número total de IES (INEP, 2009).

Tabela 1. Evolução do número de Instituições da Educação Superior, Brasil 2005-2009

\begin{tabular}{|c|c|c|c|}
\hline \multirow{2}{*}{ Ano } & \multicolumn{2}{|c|}{ Públicas } & \multirow{2}{*}{ Privadas } \\
\cline { 2 - 3 } & Federais & Estaduais & \\
\hline 2005 & 97 & 75 & 1934 \\
\hline 2006 & 105 & 83 & 2022 \\
\hline 2007 & 106 & 82 & 2032 \\
\hline 2008 & 93 & 82 & 2016 \\
\hline 2009 & 94 & 84 & 2069 \\
\hline
\end{tabular}

Enquanto as instituições públicas são mantidas, fundamentalmente, com recursos governamentais, as instituições privadas são sustentadas, de forma muito importante, pelas anuidades pagas pelos alunos. 
No setor privado do ensino superior, a partir do final dos anos 1990, começou a forte competição entre os ofertantes, com impacto negativo acelerado sobre os preços, em razão do número de vagas disponiveis ter superado, e muito, a demanda efetiva, seja pelo aumento de oferta das instituições estabelecidas, seja pela chegada de novos ofertantes ao mercado, ou ainda pela diminuição do ritmo de crescimento do número de egressos do ensino médio (GUIMARÃES; PIRES, 2006).

A análise quantitativa e qualitativa do que é publicado nos principais periódicos de determinada área do conhecimento é uma das formas de avaliarmos a produção científica. Essa análise é o objeto de estudo da cienciometria, ciência que surgiu nos anos 60 (VANTI, 2005). Para estudar a produção científica, a cienciometria vale-se de indicadores bibliométricos, tais como publicação de artigos, frequência de citação dos artigos e fator de impacto dos periódicos em que os artigos são publicados. Esse instrumental analítico foi empregado para estudar a produção científica brasileira a partir da década de 70 (MOREL; MOREL, 1977).

A partir da década de 90, o grupo de Meneghini tem dado enormes contribuições sobre a avaliação da produção científica da bioquímica brasileira.

A fim de avaliar a produção e a produtividade científico-tecnológica, têm-se utilizado largamente alguns indicadores de desempenho: índice de citações, prestígio dos periódicos onde os trabalhos são publicados, peer-ranking (onde os pares são solicitados a classificar em ordem de desempenho os grupos de área de pesquisa similar). [...] O uso de indicadores múltiplos tem sido compreendido como o melhor modo de medir e refletir o desempenho científico (MENEGHINI; FONSECA, 1990, p. 629-646).

Para revelar o estado da arte de um campo de estudos é preciso medi-lo ou possuir indicadores que permitam avaliar a sua evolução. Assim, técnicas cientométricas e bibliométricas permitem uma análise quantitativa e qualitativa de áreas científicas e seus resultados. A ciência, como um amplo sistema social, tem como uma das funções 
a disseminação do conhecimento (MACIAS-CHAPULA, 1998). Portanto, uma análise adequada da atividade social da ciência pode ser entendida como uma significante contribuição.

Os indicadores da produção científica estão no centro dos debates, seja sob a perspectiva das relações entre o avanço da ciência e da tecnologia, seja avaliando o progresso econômico e social (Ibidem). Estudos bibliométricos sobre a atividade científica vêm sendo realizados em diversas áreas, como, por exemplo, em marketing (VIEIRA, 2003), em recursos humanos (TONELLI et al., 2003), responsabilidade social (MORETTI; FIGUEIREDO, 2007) e muitos outros. Recentemente, Leta e De Meis (1996) também utilizaram dados cientométricos para traçar o perfil das diferentes áreas do conhecimento no Brasil durante os anos 1981-1993. Em relação à produção científica de áreas específicas, a Bioquímica é bastante estudada no Brasil.

Referindo-se especificamente à pós-graduação da Bioquímica no Brasil anteriormente a 1965 (MOTOYAMA, 1988), os dados estatísticos divulgados pela Capes são as fontes de maior utilidade para se traçar uma panorâmica do desenvolvimento dos PPG dessa área no País a partir dos anos 60.

No Brasil, anteriormente ao século XX, trabalhos científicos em Biologia foram os desenvolvidos pelo naturalista Fritz Muller e pelo fisiologista João Batista de Lacerda Martins (AZEVEDO, 1994).

A atividade de investigação científica em Bioquímica foi realizada inicialmente em algumas poucas instituições, geralmente localizadas no eixo Rio (Instituto Manguinhos, Instituto de Biofísica da Universidade do Brasil) - São Paulo (Instituto Butantan, Instituto Biológico, Departamento de Química da USP, Departamento de Química Fisiológica da Faculdade de Medicina da USP) - Belo Horizonte (Faculdade de Medicina da Universidade de Minas Gerais). Há também registros de atividades importantes na Faculdade de Medicina da Universidade do Paraná.

Em algumas dessas instituições, a atividade de pós-graduação começou a florescer. Teses de doutorado e de livre docência foram 
defendidas na Faculdade de Medicina da Universidade de Minas Gerais (a partir dos anos 40, orientadas por Baeta Vianna), na Faculdade de Medicina da Universidade do Paraná (sete teses de doutorado e duas de livre docência, nas décadas de 40 e 50) e no Instituto de Biofísica da Universidade do Brasil (um total de 13 teses, entre 1946 e 1959).

Foi no Departamento de Química da USP que a pós-graduação em Bioquímica se instalou com maior vigor, a partir dos anos 70 (SCHWARTZMAN, 1979). A criação da Universidade de São Paulo, ocorrida em 1934, é um dos mais importantes marcos do ensino superior brasileiro. Pela primeira vez, o País via nascer uma instituição de ensino superior que associava ensino e pesquisa e que se orientava para o progresso da ciência.

No final da década de 60, quando a pós-graduação foi oficialmente instituída no País, existiam cinco cursos de pós-graduação em Bioquímica, regularmente organizados. No ano de 2001, existiam 13 programas de pós-graduação em Bioquímica no Brasil, sendo que a maioria já se concentrava em dois estados da Federação - Rio de Janeiro e São Paulo.

Atualmente, existem 34 cursos de pós-graduação em Bioquímica no Brasil, sendo 16 de mestrado, 15 de doutorado e três de mestrado profissionalizante (CAPES, 2011).

A partir dessas considerações, o objetivo geral desta pesquisa é caracterizar os professores/pesquisadores de Bioquímica das IES públicas federais, públicas estaduais e privadas do estado do Rio Grande do Sul, além de quantificar e analisar a sua produção científica e o número de dissertações e teses orientadas, representando a formação de recursos humanos.

\section{Metodologia}

Foram analisadas as IES públicas estadual e privadas sediadas no Rio Grande do Sul que possuem departamentos ou setores de Bioquímica. Tornaram-se alvo de estudo as seguintes instituições. 
Tabela 2. IES no RS

\section{INSTITUICÕES NO RS}

\section{IES PÚBLICAS}

UNIVERSIDADE ESTADUAL DO RIO GRANDE DO SUL - UERGS

FUNDAÇÃO UNIVERSIDADE DO RIO GRANDE - Furg

UNIVERSIDADE FEDERAL DE CIÊNCIAS DA SAÚDE DE PORTO ALEGRE - UFCSPA

UNIVERSIDADE FEDERAL DE PELOTAS - UFPel

UNIVERSIDADE FEDERAL DO RIO GRANDE DO SUL - UFRGS

UNIVERSIDADE FEDERAL DE SANTA MARIA - UFSM

UNIVERSIDADE FEDERAL DO PAMPA - Unipampa

\section{IES PRIVADAS}

CENTRO UNIVERSITÁRIO FEEVALE - Feevale

FACULDADE DA SERRA GAÚCHA - FSG

CENTRO UNIVERSITÁRIO METODISTA - IPA

PONTIFÍCIA UNIVERSIDADE DE PELOTAS - PUC-RS

UNIVERSIDADE CATÓLICA DE PELOTAS - UCPel

UNIVERSIDADE DE CAXIAS DO SUL - UnisC

UNIVERSIDADE LUTERANO DO BRASIL - UIbra

CENTRO UNIVERSITÁRIO FRANCISCANO - Unifra

UNIVERSIDADE REGIONAL DO NOROESTE DO ESTADO DO RIO GRANDE DO SUL -

Unijuí

CENTRO UNIVERSITÁRIO LA SALLE - Unilasalle

UNIVERSIDADE DE SANTA CRUZ DO SUL - UnisC

UNIVERSIDADE DO VALE DO RIO DOS SINOS - Unisinos

UNIVERSIDADE REGIONAL INTEGRADA DO ALTO URUGUAI E DAS MISSÕES - URI

O período dos dados analisados foi de 1999 a 2009; o estudo inclui 212 professores/pesquisadores.

Setores ou departamentos foram contatados, por telefone e correio eletrônico, e informações foram adquiridas por meio de responsáveis ou representantes dos departamentos/setores, a quem foram solicitadas as listagens de professores em atividade no período de 1999 a 2009.

De cada setor ou departamento, levantaram-se os nomes dos professores que atuam ou atuavam no período estudado, obtendo-se as informações.

Foram extraídos do currículo Lattes os dados individuais do perfil de cada professor constante do estudo (sexo, titulação - graduação, mestrado, doutorado, pós-doutorado), destacando as instituições de ensino superior onde foi feita a formação, bem como os orientadores de mestrado, doutorado e pós-doutorado, a produção científica 
individual e a formação de recursos humanos. Depois de identificado o perfil, os dados foram agrupados por IES. Para a produção científica, analisou-se a totalidade de artigos completos publicados indexados e não indexados pelos professores/pesquisadores enquanto estiveram vinculados aos departamentos/setores de Bioquímica no período do estudo. Para que não houvesse repetições, os artigos científicos foram separados por ano e por professores, eliminando as sobreposições. Consideraram-se como produção científica os artigos publicados por revistas indexadas no banco de dados do Institute for Scientific Information (ISI) e em revistas não indexadas. Verificaram-se ainda todas as orientações de mestrado e doutorado concluídas no período.

\section{Resultados e discussão}

Inicialmente, caracterizamos o perfil de 212 professores pertencentes a departamentos e setores de Bioquímica das instituições públicas e privadas de ensino superior no RS, em atividade no período de 1999-2009. Observamos (Tabela 3) que a UERGS possui o maior número de professores atuando no setor de Bioquímica, provavelmente por que há uma alta rotatividade em relação às demais instituições pesquisadas. De maneira geral, apesar de detalhes específicos, há uma distribuição mais equilibrada entre os gêneros nas instituições federais, enquanto há predominância de mulheres nas instituições privadas.

Tabela 3. Gênero dos professores/pesquisadores nos departamentos/ setores das IES

\begin{tabular}{|c|c|c|c|}
\hline \multirow{2}{*}{\multicolumn{2}{|c|}{ Instituições }} & \multicolumn{2}{|c|}{ Sexo $n(\%)$} \\
\hline & & $F$ & $M$ \\
\hline \multirow{7}{*}{ 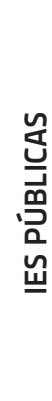 } & UERGS (50) & $30(60)$ & $20(40)$ \\
\hline & Furg $(04)^{*[1]}$ & 0 & $4(100)$ \\
\hline & UFCSPA (06) & $6(100)$ & 0 \\
\hline & UFPel $(13) *[5]$ & $4(31)$ & 9 (69) \\
\hline & UFRGS (33) & $17(51,5)$ & $16(48,5)$ \\
\hline & UFSM (11) & $6(54,5)$ & $5(45,5)$ \\
\hline & Unipampa (07) & $4(57,1)$ & $3(42,9)$ \\
\hline
\end{tabular}




\begin{tabular}{|c|c|c|c|}
\hline \multirow{2}{*}{ Instituições } & \multirow{2}{*}{ Instituições } & \multicolumn{2}{|c|}{ Sexo $n(\%)$} \\
\hline & & $\mathrm{F}$ & M \\
\hline \multirow{13}{*}{ 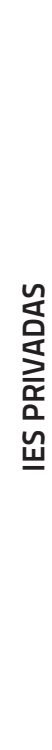 } & Feevale (05) & $5(100)$ & 0 \\
\hline & FSG (03) & $2(66,6)$ & $1(33,4)$ \\
\hline & IPA (08) & $5(62,5)$ & $3(37,5)$ \\
\hline & PUC-RS (08) & $5(62,5)$ & $3(37,5)$ \\
\hline & UCPel (05) & $3(60)$ & $2(40)$ \\
\hline & UCS (02) & $2(100)$ & 0 \\
\hline & Ulbra (10) & $9(90)$ & $1(10)$ \\
\hline & Unifra (15) & $8(53,4)$ & $7(46,6)$ \\
\hline & Unijuí(06) & $6(100)$ & 0 \\
\hline & Unilasalle (03) & $3(100)$ & 0 \\
\hline & Unisc (08) & $5(62,5)$ & $3(37,5)$ \\
\hline & Unisinos (07) & $4(57,1)$ & $3(42,9)$ \\
\hline & URI (08) & $4(50)$ & $4(50)$ \\
\hline
\end{tabular}

Com relação à formação graduada dos professores/ pesquisadores, observamos a presença de profissionais da área da saúde, químicos e biólogos (Tabela 4). Nas universidades públicas, notamos que na UFRGS e na UFSM há mais farmacêuticos, que atingem quase a metade dos professores. Na UFRGS, a segunda maior incidência é de médicos e na UFSM de biólogos. Na UERGS predominam os químicos. Há maior número de biólogos na UFCSPA e na FURG. Na UFPel e na Unipampa existe predominância de agrônomos, enfermeiros e médicos veterinários. Nas instituições privadas, tendem a predominar os farmacêuticos. 
Tabela 4: Formação graduada dos professores/pesquisadores em Bioquímica no RS

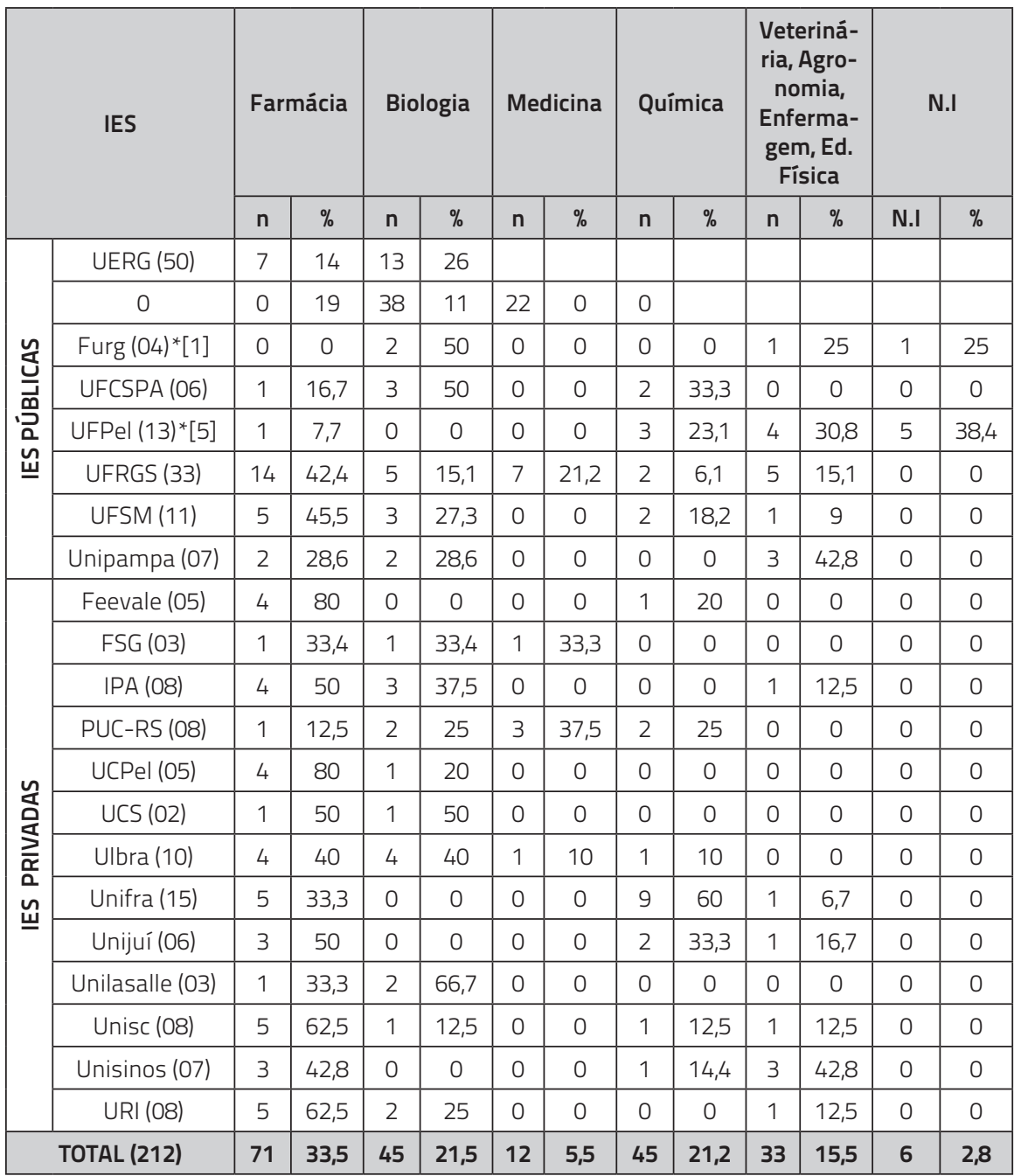

Obs.: 0 * equivale à instituição (número de professores/pesquisadores no período estudado). 0 [ ] a instituições (dep./setor) com professores que não possuem Currículo Lattes.

A maior parte dos professores/pesquisadores de todas as instituições tiveram formação pós-graduada (Tabela 5). A maioria das IES tem a totalidade (ou quase) dos professores/pesquisadores pós-graduados, com exceção da UFPel (o que pode estar relacionado à presença de professores mais antigos, cujos CV Lattes não foram sequer encontrados). Nas IES públicas, particularmente na UFRGS, UFSM e Unipampa, mais da metade dos professores/pesquisadores possui pós-doutorado. Entre os professores da UERGS (em torno de $25 \%$ ) e de várias instituições privadas, há uma percentagem importante de pós-doutores. 
Tabela 5. Formação pós-graduada dos professores/pesquisadores nos departamentos/setores das IES

\begin{tabular}{|c|c|c|c|c|c|c|c|}
\hline \multirow{2}{*}{\multicolumn{2}{|c|}{ IES }} & \multicolumn{2}{|c|}{ Com PG } & \multicolumn{2}{|c|}{ Doutorado } & \multicolumn{2}{|c|}{ Pós-Doutorado } \\
\hline & & $\mathrm{n}$ & $\%$ & $\mathrm{n}$ & $\%$ & $\mathrm{n}$ & $\%$ \\
\hline \multirow{7}{*}{ 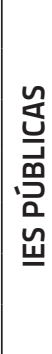 } & UERGS (50) & 49 & 98 & 40 & 80 & 14 & 27,4 \\
\hline & Furg $(04) *[1]$ & 3 & 75 & 3 & 75 & 0 & 0 \\
\hline & UFCSPA (06) & 6 & 100 & 6 & 100 & 0 & 0 \\
\hline & UFPel (13)8[5] & 6 & 46,4 & 5 & 38,4 & 1 & 7,7 \\
\hline & UFRGS (33) & 33 & 100 & 33 & 100 & 18 & 54,5 \\
\hline & UFSM (11) & 11 & 100 & 11 & 100 & 6 & 54,5 \\
\hline & Unipampa (07) & 7 & 100 & 7 & 100 & 4 & 57,1 \\
\hline \multirow{13}{*}{ 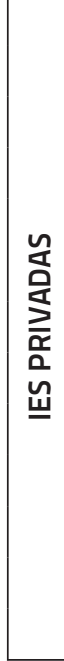 } & Feevale (05) & 5 & 100 & 5 & 100 & 1 & 20 \\
\hline & FSG (03) & 3 & 100 & 2 & 66,6 & 1 & 33,3 \\
\hline & IPA (08) & 7 & 87,5 & 6 & 75 & 2 & 25 \\
\hline & PUC-RS (08) & 8 & 100 & 7 & 87,5 & 1 & 12,5 \\
\hline & UCPel (05) & 4 & 80 & 4 & 80 & 1 & 20 \\
\hline & UCS (02) & 2 & 100 & 2 & 100 & 0 & 0 \\
\hline & Ulbra (10) & 10 & 100 & 10 & 100 & 3 & 30 \\
\hline & Unifra (15) & 13 & 100 & 11 & 73,4 & 1 & 6,6 \\
\hline & Unijuí (06) & 6 & 100 & 5 & 83,4 & 1 & 16,7 \\
\hline & Unilasalle (03) & 3 & 100 & 3 & 100 & 0 & 0 \\
\hline & Unisc (08) & 8 & 100 & 5 & 62,5 & 0 & 0 \\
\hline & Unisinos (07) & 7 & 100 & 6 & 85,7 & 1 & 16,7 \\
\hline & URI (08) & 7 & 87,5 & 7 & 87,5 & 1 & 12,5 \\
\hline \multicolumn{2}{|c|}{ TOTAL (212) } & 199 & 94 & 178 & 84 & 56 & 26,4 \\
\hline
\end{tabular}

Obs.: 0 * equivale à instituição (número de professores/pesquisadores no período estudado). 0 [ ] a instituições (dep./setor) com professores que não possuem Currículo Lattes.

Grande parte dos professores/pesquisadores se doutorou no PPG em Bioquímica da UFRGS, programa mais antigo na área no estado. Dos profissionais que fizeram pós-doutorado, concentrados mais nas instituições federais (UFRGS, UFSM e Unipampa), a maioria se formou no exterior (Tabelas $6 \mathrm{~A}$ e B). 
Tabela 6A. Local do doutorado dos professores/pesquisadores nos departamentos/setores das IES

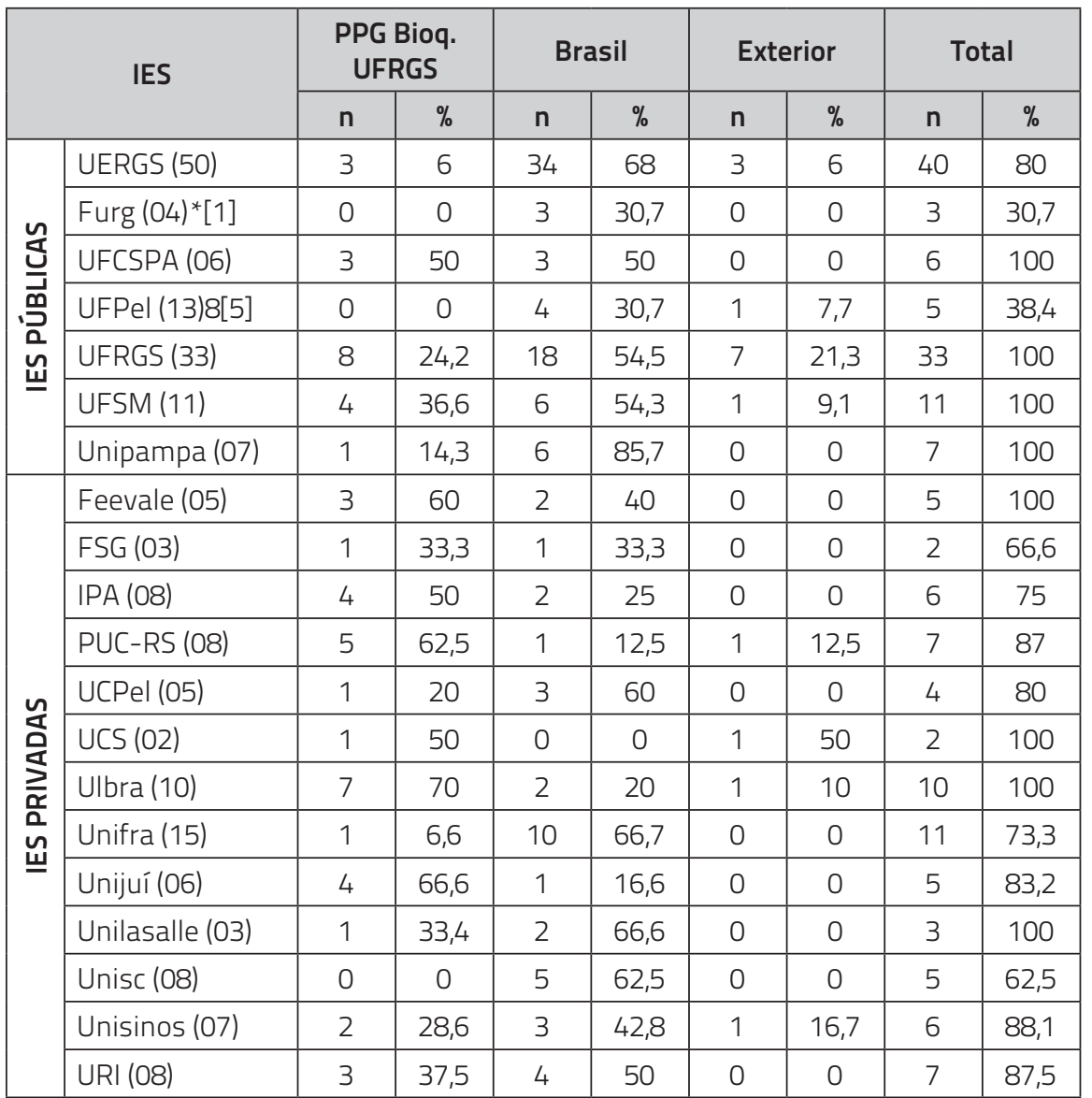

Obs.: 0 * equivale à instituição (número de professores/pesquisadores no período estudado). O [ ] a instituições (dep./setor) com professores que não possuem Currículo Lattes. 
Tabela 6B. Local do pós-doutorado dos professores/pesquisadores nos departamentos/setores das IES

\begin{tabular}{|c|c|c|c|c|c|c|c|c|c|}
\hline & \multirow{2}{*}{ IES } & \multicolumn{2}{|c|}{$\begin{array}{l}\text { PPG Bioq. } \\
\text { UFRGS }\end{array}$} & \multicolumn{2}{|c|}{ Brasil } & \multicolumn{2}{|c|}{ Exterior } & \multicolumn{2}{|c|}{ Total } \\
\hline & & $n$ & $\%$ & $\mathrm{n}$ & $\%$ & $\mathrm{n}$ & $\%$ & $n$ & $\%$ \\
\hline \multirow{7}{*}{ 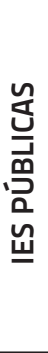 } & UERGS (50) & 1 & 2 & 9 & 18 & 4 & 8 & 14 & 28 \\
\hline & Furg $(04) *[1]$ & 0 & 0 & 0 & 0 & 0 & 0 & 0 & 0 \\
\hline & UFCSPA (06) & 0 & 0 & 0 & 0 & 0 & 0 & 0 & 0 \\
\hline & UFPel (13)8[5] & 0 & 0 & 1 & 7,7 & 0 & 0 & 1 & 7,7 \\
\hline & UFRGS (33) & 0 & 0 & 1 & 3 & 18 & 54,5 & 19 & 57,5 \\
\hline & UFSM (11) & 0 & 0 & 3 & 27,3 & 3 & 27,3 & 6 & 54,6 \\
\hline & Unipampa (07) & 0 & 0 & 2 & 28,6 & 2 & 28,6 & 4 & 57,2 \\
\hline \multirow{13}{*}{$\begin{array}{l}\text { 号 } \\
\text { 号 } \\
\text { 品 } \\
\text { 品 }\end{array}$} & Feevale (05) & 0 & 0 & 1 & 20 & 0 & 0 & 1 & 20 \\
\hline & FSG (03) & 0 & 0 & 1 & 33,3 & 0 & 0 & 1 & 33,3 \\
\hline & IPA (08) & 1 & 12,5 & 1 & 12,5 & 0 & 0 & 2 & 25 \\
\hline & PUC-RS (08) & 0 & 0 & 0 & 0 & 1 & 12,5 & 1 & 12,5 \\
\hline & UCPel (05) & 1 & 20 & 0 & 0 & 0 & 0 & 1 & 20 \\
\hline & UCS (02) & 0 & 0 & 0 & 0 & 0 & 0 & 0 & 0 \\
\hline & Ulbra (10) & 0 & 0 & 1 & 10 & 2 & 20 & 3 & 30 \\
\hline & Unifra (15) & 0 & 0 & 0 & 0 & 1 & 6,7 & 1 & 6,7 \\
\hline & Unijuí (06) & 0 & 0 & 0 & 0 & 1 & 16,6 & 1 & 16,6 \\
\hline & Unilasalle (03) & 0 & 0 & 0 & 0 & 0 & 0 & 0 & 0 \\
\hline & Unisc (08) & 0 & 0 & 0 & 0 & 0 & 0 & 0 & 0 \\
\hline & Unisinos (07) & 0 & 0 & 1 & 14,3 & 0 & 0 & 1 & 14,3 \\
\hline & URI (08) & 0 & 0 & 1 & 12,5 & 0 & 0 & 1 & 12,5 \\
\hline
\end{tabular}

Obs.: 0 * equivale à instituição (número de professores/pesquisadores no período estudado). 0 [ ] a instituições (dep./setor) com professores que não possuem Currículo Lattes.

Dentre os 212 professores que atuam nas 20 IES estudadas, apenas 24 estão alocados nos respectivos departamentos/setores de Bioquímica há mais de 20 anos, sendo que praticamente a totalidade deles é da UFRGS (Tabela 7). Isso pode ser explicado pela maior antiguidade do Departamento de Bioquímica, pelo Instituto de Ciências Básicas e da Saúde da UFRGS, pela manutenção de seus pesquisadores nessa atividade e pela criação mais recente de setores de Bioquímica na maioria das outras instituições estudadas. Com 10 a 20 anos de atuação, encontram-se 37 professores, chamando a atenção para o fato que, nesse grupo, está a maior proporção de professores da UFSM e Ulbra. Grande parte dos outros 144 professores está há menos de 10 anos em atividade no local de trabalho e em cargos atuais, distribuídos em todas as instituições em estudo. 
Tabela 7. Tempo de atuação dos professores/pesquisadores nos departamentos/setores das IES

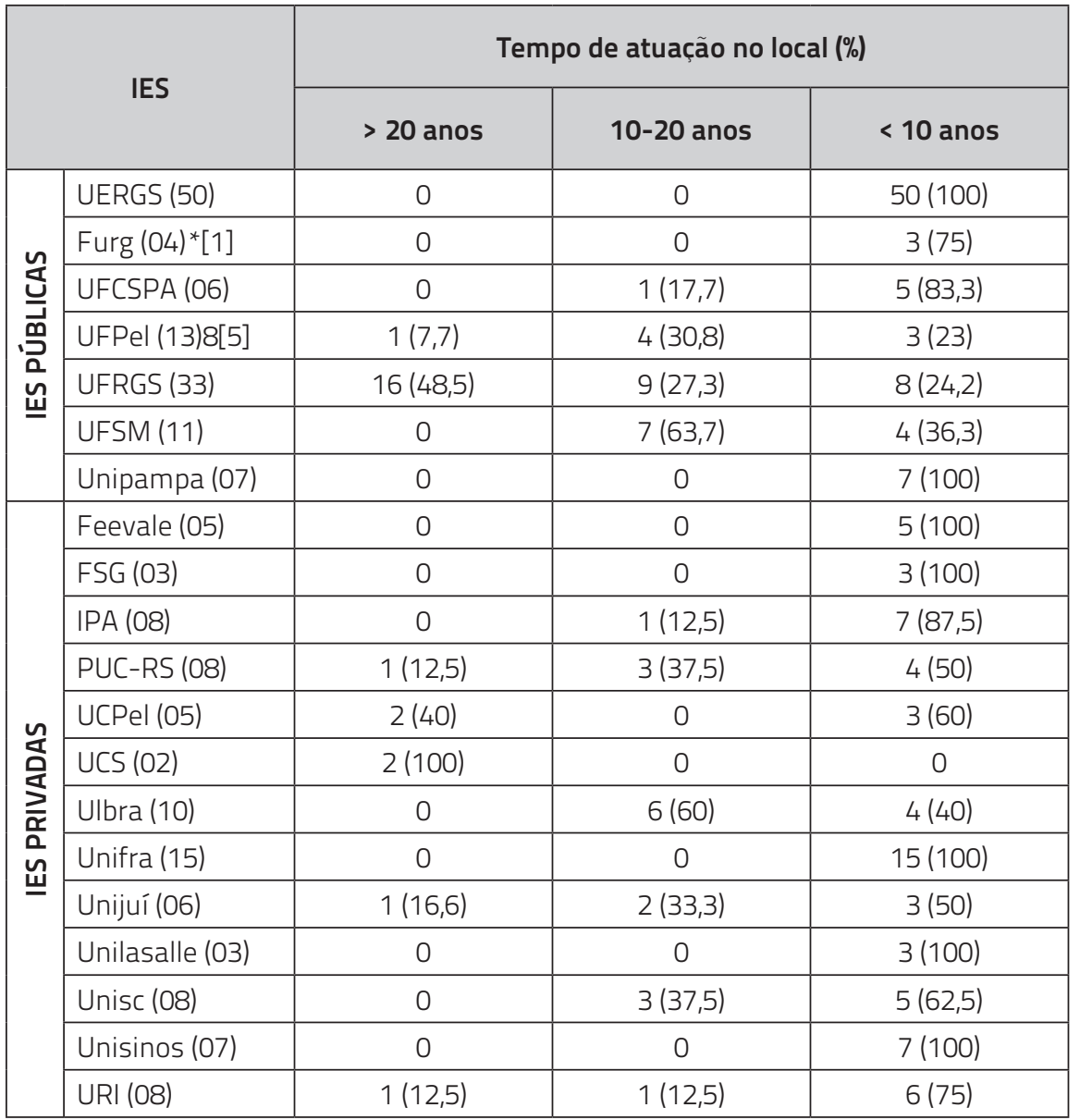

Obs.: 0 * equivale à instituição (número de professores/pesquisadores no período estudado). O [ ] a instituições (dep./setor) com professores que não possuem Currículo Lattes.

Entre 1999 e 2009, percebe-se que a maior produção científica das universidades públicas veio da UFRGS (Tabela 8A), o que pode estar relacionado ao número de professores, tempo de atuação e ao fato de o PPG em nivel de mestrado e doutorado nessa instituição ser o mais antigo. Destaca-se também a UFSM, que possui PPG em nível de mestrado e doutorado diretamente vinculado à área de Bioquímica. Apesar do PPG em Bioquímica da Unipampa ser recente (2009), sua produção é relevante (alto número de publicações, todas em revistas indexadas). Nas IES privadas, observamos que a PUCRS e a Ulbra se sobressaem comparativamente às demais instituições privadas. 
Tabela 8A. Produção científica e orientações de mestrado e doutorado entre 1999-2009

\begin{tabular}{|c|c|c|c|c|c|}
\hline & IES & $\begin{array}{c}\mathrm{N}^{\circ} \text { de } \\
\text { Professores }\end{array}$ & $\begin{array}{c}\text { Produção Total } \\
\text { 99-2009 (\% artigos } \\
\text { indexados) }\end{array}$ & $\begin{array}{l}\text { Orientações } \\
\text { Mestrado }\end{array}$ & $\begin{array}{c}\text { Orientações } \\
\text { Doutorado }\end{array}$ \\
\hline \multirow{7}{*}{ 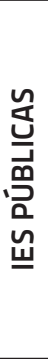 } & UERGS & 50 & $93(63,4)$ & 6 & 0 \\
\hline & Furg & 4 & $91(76,9)$ & 14 & 3 \\
\hline & UFCSPA & 6 & $39(64,1)$ & 0 & 0 \\
\hline & UFPel & 13 & $49(32,6)$ & 9 & 1 \\
\hline & UFRGS & 33 & $1222(94,3)$ & 240 & 142 \\
\hline & UFSM & 11 & $590(95,6)$ & 134 & 46 \\
\hline & Unipampa & 7 & 41 (100) & 2 & 0 \\
\hline \multirow{13}{*}{ 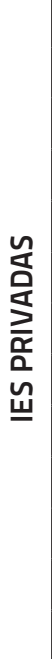 } & Feevale & 5 & $44(54,5)$ & 0 & 0 \\
\hline & FSG & 3 & 07 (100) & 0 & 0 \\
\hline & IPA & 8 & $53(62,4)$ & 0 & 0 \\
\hline & PUC-RS & 8 & $232(95,7)$ & 42 & 16 \\
\hline & UCPel & 5 & $38(26,3)$ & 0 & 0 \\
\hline & UCS & 2 & $66(68,2)$ & 16 & 3 \\
\hline & Ulbra & 10 & $224(81,7)$ & 56 & 7 \\
\hline & Unifra & 15 & $61(54,1)$ & 5 & 0 \\
\hline & Unijuí & 6 & $26(65,3)$ & 5 & 0 \\
\hline & Unilasalle & 3 & $14(57,1)$ & 0 & 0 \\
\hline & Unisc & 8 & $42(40,5)$ & 2 & 0 \\
\hline & Unisinos & 7 & $11(91)$ & 0 & 0 \\
\hline & URI & 8 & $61(52,4)$ & , & 0 \\
\hline
\end{tabular}

Obs.: 0 * equivale à instituição (número de professores/pesquisadores no período estudado). 0 [ ] a instituições (dep./setor) com professores que não possuem Currículo Lattes.

Baseado no Science Citation Index (SCI), que é a base de dados do ISI, divulgado pelo Journal Citation Report (JCR), a grande maioria das publicações científicas de todas as IES públicas (com exceção da UFPel) é indexada. O mesmo se observa nas IES privadas, com exceção da UCPel e Unisc. Esses dados podem refletir a vocação científica dos diferentes centros, levando à opção por publicações em periódicos indexados ou não.

Quanto à orientação de pós-graduação, a UFRGS (M: 240 e D: 142) e UFSM (M: 134 e D: 46) se destacam, acompanhando a produção científica dos professores. 
Nas outras instituições, a relação da produção científica com a orientação é difícil de ser avaliada, pois, como já citado, não há, nessas instituições, PPGs diretamente vinculados ao setor; as orientações, portanto, estavam vinculavas a outros PPGs da mesma IES ou de outras.

Entre as IES privadas, o número de dissertações de mestrado garante destaque à Ulbra (M: 56 e D: 7), PUCRS (M: 42 e D:16) e UCS (M: 16 e D: 3) (Tabela 8A).

Fizemos a análise da produção científica e de suas correlações no último ano do estudo (2009) (Tabela 8B). A relação artigo indexado/ docente nas públicas foi elevada na UFSM (9,8), na Furg (3,7), na UFRGS $(3,2)$ e na Unipampa (2,8). Na UERGS, UFCSPA e UFPel, essa relação não chega a um. Dentre as IES privadas, podemos destacar a UCS (4,5), a PUC-RS $(3,6)$ e a Ulbra $(2,3)$.

Tabela 8B. Produção científica, orientação de mestrado e doutorado 2009

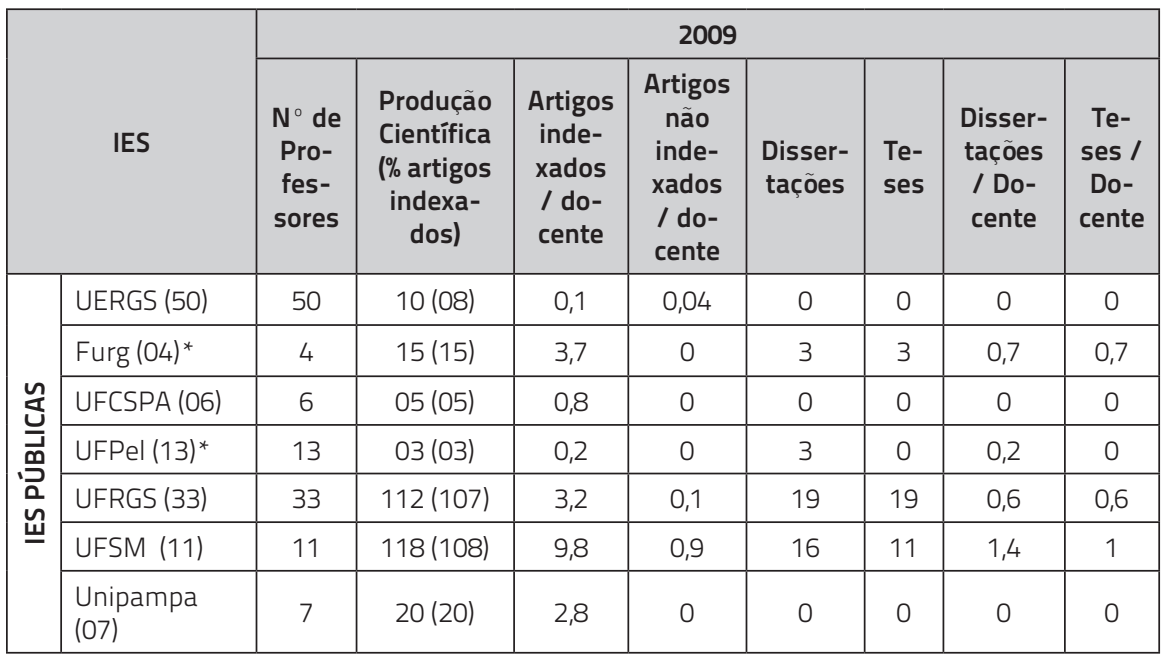




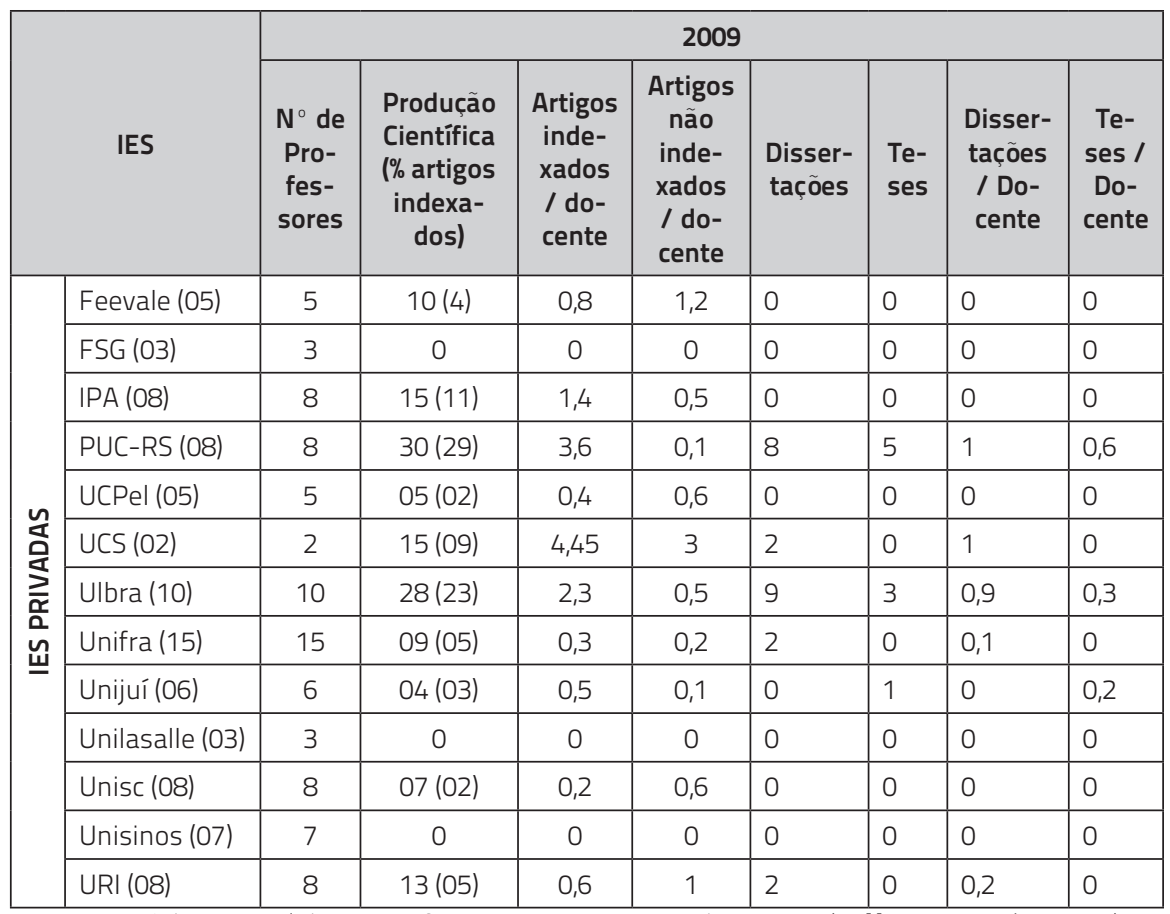

Obs.: 0 * equivale à instituição (número de professores/pesquisadores no período estudado). 0 [ ] a instituições (dep./ setor) com professores que não possuem Currículo Lattes.

Quando analisamos o índice de artigos não indexados/docente, percebemos que ele é muito baixo em todas as IES públicas. Nas IES privadas, embora esse índice seja também baixo, ele é superior ao de artigos indexados/docentes (Feevale, UCPel, Unisc e URI).

Quando analisamos os índices de dissertações/docente e teses/docente, destaca-se a UFSM entre as IES públicas, seguida pela Furg e UFRGS. Nas IES privadas, esse índice destaca a PUC-RS e Ulbra (e a UCS na dissertação).

Nosso grupo publicou recentemente (BERTI et al., 2010) a inexistência de PPG em Bioquímica nas instituições federais UFCSPA, UFPel e Furg, onde alguns professores de Bioquímica orientam PPG em outras áreas. Isso poderia explicar o baixo índice de dissertações e teses por docente.

Muitos dos professores que atuam na UFSM foram formados pelos professores da UFRGS e atualmente formam professores da Unipampa; talvez essa seja a razão de perfis parecidos de produção e 
vocação científica entre as três IES. Alguns dos professores da UFCSPA também foram orientados pelos professores da Bioquímica da UFRGS, mas como sua IES não possui PPG em Bioquímica, eles têm dificuldade em publicar e orientar.

Podemos observar, em resumo, que a maioria $(96 \%)$ dos professores/pesquisadores em Bioquímica de todas as instituições, públicas ou privadas, do RS possui doutorado $(86,4 \%)$, e um número significativo realizou pós-doutorado (27,2\%), atestando uma vocação para a ciência, mas nem todos encontram condições de seguir atividades de pesquisa naquelas instituições que os acolhem. Isso pode ser devido ao perfil e/ou vocação das IES/RS.

Recebido em 28/07/2011

Aprovado em 25/03/2013

\section{Agradecimentos}

À Capes, ao Instituto Nacional de Ciência e Tecnologia em Excitotoxicidade e Neuroproteção (CNPq) e à Fundação de Amparo à Pesquisa do Estado do Rio Grande do Sul.

\section{Referências bibliográficas}

AZEVEDO, F. As Ciências no Brasil. 2. ed. Rio de Janeiro: Editora da Universidade Federal do Rio de Janeiro, 1994.

BERTI, L. C.; et al. Produção Científica e Formação de Recursos Humanos na área de Bioquímica em Instituições Federais do Rio Grande do Sul: Fomento Estadual. Química Nova, v. 33, n. 3, p. 765-771, 2010.

CAPES - Coordenação de Aperfeiçoamento de Pessoal de Nivel Superior. Cursos recomendados e reconhecidos por área de avaliação.

Disponivel em: http:/ / www.capes.gov.br. Acesso em: 11 jul. 2011.

GUIMARÃES, A. S.; PIRES, V. Ensino Superior no Brasil: mercado, regulação e estratégias. Eccos, v. 8, n. 2, p. 227-247, 2006. 
INEP - Instituto Nacional de Estudos e Pesquisas Educacionais. Resumo técnico do censo da educação superior de 2007. Brasília: Inep/ MEC, 2009.

LEITE, J. L.; et al. Os projetos de pesquisa em enfermagem no CNPq: seu percurso, suas temáticas, suas aderências. Revista Brasileira de Enfermagem, v. 54, n. 1, p. 81-97, 2001.

LETA, J.; DE MEIS, L. A profile of science in Brazil. Scientometrics, v. 35, p. 33-44, 1996.

MACIAS-CHAPULA, C. O papel da informetria e da cienciometria e sua perspectiva nacional e internacional. Ciência da Informação, v. 27, n. 2, p. 134-140, 1998.

MENDES, I. A. C. Pesquisa em enfermagem: impacto na prática. São Paulo: Edusp, 1991.

MENEGHINI, R.; FONSECA, L. Índices alternativos de avaliação da produção científica em bioquímica no Brasil. Ciência e Cultura, São Paulo, v. 42, n. 9, p. 629-46, 1990.

MOREL, R. L.; MOREL, C. M. Um estudo sobre a produção científica brasileira, segundo os dados do Institute for Scientific Information. Ciência da Informação, v. 7, p. 79-83, 1977.

MORETTI, S.; FIGUEIREDO, J. Análise bibliométrica da produção sobre responsabilidade social das empresas no EnANPAD: evidências de um discurso monológico. Revista de Gestão Social e Ambiental, v. 1, n. 3, p. 21-38, 2007.

MOTOYAMA, S. A História das Ciências no Brasil. Apontamentos para uma análise crítica. São Paulo: Edusp, 1988.

RUTHERFORD, F. J.; ALGREEN, A. Science for all Americans. Nova York: Oxford, 1990. 
SCHWARTZMAN, S. Formação da Comunidade Científica no Brasil. São Paulo: Editora Nacional, 1979.

TONELLI, M.; et al. Produção acadêmica em recursos humanos no Brasil: 1991-2000. Revista de Administração de Empresas, v. 43, n. 1, p. 105-122, 2003.

UNESCO. World Conference on Science. Science for the twenty-first century. Paris: UNESCO, 2000.

VANTI, N. Os links e os estudos webométricos. Ciência da Informação, v. 34, n. 1, p.78-88, 2005.

VIEIRA, F. Narciso sem espelho: a publicação brasileira de marketing. Revista de Administração de Empresas, v. 43, n. 1, p. 81-90, 2003.

ZANCAN, G. T. Educação Científica: Uma prioridade nacional. São Paulo em Perspectiva, v. 14, p. 3-7, 2002. 